\title{
The genome and transcriptome of the Phalaenopsis yield insights into floral organ development and flowering regulation
}

Jian-Zhi Huang, Chih-Peng Lin, Ting-Chi Cheng, Ya-Wen Huang, Yi-Jung Tsai, Shu-Yun Cheng, Yi-Wen Chen, Chueh-Pai Lee, WanChia Chung, Bill Chia-Han Chang, Shih-Wen Chin, Chen-Yu Lee, Fure-Chyi Chen

Phalaenopsis orchid is an important potted flower with high economic value around the world. We report the $3.1 \mathrm{~Gb}$ draft genome assembly of an important winter flowering Phalaenopsis 'KHM190' cultivar. We generated 89.5 Gb RNA-seq and 113 million sRNA-seq reads to use these data to identify 41,153 protein-coding genes and 188 miRNA families. We also generated a draft genome for Phalaenopsis pulcherrima 'B8802', a summer flowering species, via resequencing. Comparison of genome data between the two Phalaenopsis cultivars allowed the identification of 691,532 single-nucleotide polymorphisms. In this study, we reveal the key role of PhAGL6b in the regulation of labellum organ development involves alternative splicing in big lip mutant. Petal or sepal overexpressing PhAGL6b lead to the conversion into lip-like structure. We also evidenced the gibberellin pathway that regulates the expression of flowering time genes during the reproductive phase change induced by cool temperature. Our work thus depicted a valuable resource for the flowering control, flower architecture development, and breeding of the Phalaenopsis orchids. 
1 The genome and transcriptome of the Phalaenopsis yield insights into floral 2 organ development and flowering regulation

3

4
Jian-Zhi Huang $^{1 *}$, Chih-Peng Lin ${ }^{2,4^{*}}$, Ting-Chi Cheng ${ }^{1}$, Ya-Wen Huang ${ }^{1}$,Yi-Jung Tsai ${ }^{1}$, Shu-Yun Cheng ${ }^{1}$, Yi-Wen Chen ${ }^{1}$, Chueh-Pai Lee ${ }^{2}$, Wan-Chia Chung ${ }^{2}$, Bill Chia-Han Chang ${ }^{2,3 *}$, Shih-Wen Chin $^{1 \#}$, Chen-Yu Lee ${ }^{1 \#} \&$ Fure-Chyi Chen ${ }^{1 \#}$

${ }^{1}$ Department of Plant Industry, National Pingtung University of Science and Technology, Pingtung 91201, Taiwan

${ }^{2}$ Yourgene Bioscience, Shu-Lin District, New Taipei City 23863, Taiwan

${ }^{3}$ Faculty of Veterinary Science, The University of Melbourne, Parkville Victoria 3010 Australia

${ }^{4}$ Department of Biotechnology, School of Health Technology, Ming Chuan University, Gui Shan District, Taoyuan 333, Taiwan

${ }^{*}$ These authors contributed equally to this work.

${ }^{\#}$ Correspondence should be addressed to B-C.H.C. (bchang@yourgene.com.tw), S.-W.C.

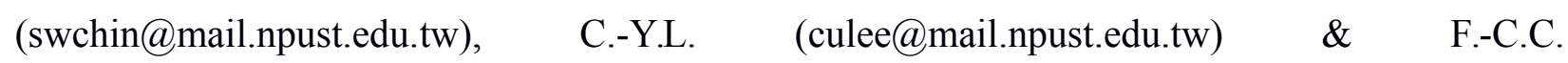

(fchen@mail.npust.edu.tw) 
35 Abstract

Phalaenopsis orchid is an important potted flower with high economic value around 


\section{Introduction}

Phalaenopsis is a genus within the family Orchidaceae and comprises approximately 66 species distributed throughout tropical Asia (Christenson 2002). The predicted Phalaenopsis genome size is approximately 1.5 gigabases $(\mathrm{Gb})$, which is distributed across 19 chromosomes (Lin et al. 2001). Phalaenopsis flowers have a zygomorphic floral structure, including three sepals (in the first floral whorl), two petals and the third petal develops into a labellum in early stage of development, which is a distinctive feature of a highly modified floral part in second floral whorl unique to orchids. The gynostemium contains the male and female reproductive organs in the center (Rudall \& Bateman 2002). In the ABCDE model, B-class genes play important role to perianth development in orchid species (Chang et al. 2010; MondragonPalomino \& Theissen 2011; Tsai et al. 2004). In addition, PhAGL6a and PhAGL6b, expressed specifically in the Phalaenopsis labellum, were implied to play as a positive regulator of labellum formation (Huang et al. 2015; Su et al. 2013). However, the relationship between the function of genes involved in floral-organ development and morphological features remains poorly understood.

Phalaenopsis orchids are produced in large quantity annually and are traded as the most important potted plants worldwide. During greenhouse production of young plants, the high temperature $>28^{\circ} \mathrm{C}$ was routinely used to promote vegetative growth and inhibit spike initiation (Blanchard \& Runkle 2006). Conversely, a lower ambient temperature $\left(24 / 18^{\circ} \mathrm{C}\right.$ day/night) is used to induce spiking (Chen et al. 2008) to produce flowering plants. Spike induction in Phalaenopsis orchid by this cool temperature is the key to precisely control its flowering date. Several studies have indicated that cool temperature during the night are necessary for Phalaenopsis orchids to flower (Blanchard \& Runkle 2006; Chen et al. 1994; Chen et al. 2008; Wang 1995). Despite a number of expressed sequence tags (ESTs), RNA-seqs and sRNA-seqs from several tisues of Phalaenopsis have been reported and deposited in GenBank or OrchidBase (An \& Chan 2012; An et al. 2011; Hsiao et al. 2011; Su et al. 2011), only a few flowering related genes or miRNAs have been identified and characterized. Besides, the clues to the spike initiation during reproductive phase change in the shorten stem, which may produce signals related to flowering during cool temperature induction, have not been dealt with. So far, the molecular mechanisms leading to spiking of Phalaenopsis has yet to be elucidated.

Here we report a high-quality genome and transcriptomes (mRNAs and small RNAs) of Phalaenopsis Brother Spring Dancer 'KHM190', a winter flowering hybrid with spike formation in response to low temperature. We also provide resequencing data for summer flowering species P. pulcherrima 'P8802'. Our comprehensive genomic and transcriptome analyses provide valuable insights into the molecular mechanisms of important biological processes such as floral organ development and flowering time regulation. 
109

110

111

112

113

114

115

116

117

118

119

120

121

122

123

124

125

126

127

128

129

130

131

132

133

134

135

136

137

138

139

140

141

142

143

144

145

\section{METHODS SUMMARY}

The genome of the Phalaenopsis Brother Spring Dancer 'KHM190' cultivar was sequenced on the Illumina HiSeq 2000 platform. The obtained data were used to assemble a draft genome sequence using the Velvet software (Zerbino \& Birney 2008). RNA-Seq and sRNA-Seq data were generated on the same platform for genome annotation and transcriptome and small RNA analyses. Repetitive elements were identified by combining information on sequence similarity at the nucleotide and protein levels and by using de novo approaches. Gene models were predicted by combining publically available Phalaenopsis RNA-Seq data and RNA-Seq data generated in this project. RNA-Seq data were mapped to the repeat masked genome with Tophat (Trapnell et al. 2009) and CuffLinks (Trapnell et al. 2012). The detailed methodology and associated references are available in the SI Appendix.

\section{Results and Discussion}

3.1 Genome sequencing and assembly. We sequenced the genome of the Phalaenopsis orchid cultivar 'KHM190' (SI Appendix, Fig. S1a) using the Illumina HiSeq 2000 platform and assembled the genome with the Velvet assembler, using $300.5 \mathrm{~Gb}$ (90-fold coverage) of filtered high-quality sequence data (SI Appendix, Table S1). This cultivar has an estimated genome size of $3.45 \mathrm{~Gb}$ on the basis of a 17-mer depth distribution analysis of the sequenced reads (SI Appendix, Fig. S2 and S3 and Table S2 and S3). De novo assembly of the Illumina reads resulted in a sequence of $3.1 \mathrm{~Gb}$, representing $89.9 \%$ of the Phalaenopsis orchid genome. Following gap closure, the assembly consisted of 149,151 scaffolds ( $\geq 1000 \mathrm{bp}$ ), with N50 lengths of $100 \mathrm{~kb}$ and $1.5 \mathrm{~kb}$ for the contigs. Approximately $90 \%$ of the total sequence was covered by 6,804 scaffolds of $>100 \mathrm{~kb}$, with the largest scaffold spanning 1.4 Mb (SI Appendix, Table S3-S5 and Dataset S17). The sequencing depth of $92.5 \%$ of the assembly was more than 20 reads (SI Appendix, Fig. S3), ensuring high accuracy at the nucleotide level. The GC content distribution in the Phalaenopsis genome was comparable with that in the genomes of Arabidopsis (2000), Oryza (2005) and Vitis (Jaillon et al. 2007) (SI Appendix, Fig. S4).

3.2 Gene prediction and annotation. Approximately $59.74 \%$ of the Phalaenopsis genome assembly was identified as repetitive elements, including long terminal repeat retrotransposons (33.44\%), DNA transposons (2.91\%) and unclassified repeats (21.99\%) (SI Appendix, Fig. S5 and Table S6). To facilitate gene annotation, we identified 41,153 high-confidence and mediumconfidence protein-coding regions with complete gene structures in the Phalaenopsis genome using RNA-Seq (114.1 Gb for a 157.6 Mb transcriptome assembly), based on 15 libraries representing four tissues (young floral organs, leaves, shortened stems and protocorm-like bodies (PLBs)) (SI Appendix, Table S7 and Dataset S18), and we used transcript assemblies of these regions in combination with publically available expressed sequence tags ( $\mathrm{Su}$ et al. 2011; Tsai et 
146

147

148

149

150

151

152

153

154

155

156

157

158

159

160

161

162

163

164

165

166

167

168

169

170

171

172

173

174

175

176

177

178

179

180

181

182

al. 2013) for gene model prediction and validation (Dataset S1-S2). We predicted 41,153 genes with an average mRNA length of 1,014 bp and a mean number of 3.83 exons per gene (Table 1 and Dataset S3). In addition to protein coding genes, we identified a total of 562 ribosomal RNAs, 655 transfer RNAs, 290 small nucleolar RNAs and 263 small nuclear RNAs in the Phalaenopsis genome (SI Appendix, Table S8). We also obtained 92,811,417 small RNA (sRNA) reads (18-27 bp), representing 6,976,375 unique sRNA tags (SI Appendix, Fig. S6 and Dataset S6-S7). A total of 650 miRNAs distributed in 188 families were identified (Dataset S8), and a total of 1,644 miRNA-targeted genes were predicted through the alignment of conserved miRNAs to our gene models (SI Appendix, Fig. S7 and Dataset S9-S10).

The Phalaenopsis gene families were compared with those of Arabidopsis (2000), Oryza (2005), and Vitis (Jaillon et al. 2007) using OrthoMCL (Li et al. 2003). We identified 41,153 Phalaenopsis genes in 15,855 families, with 8,532 gene families being shared with Arabidopsis, Oryza and Vitis. Another 5,143 families, containing 12,520 genes, were unique to Phalaenopsis (Figure 1). In comparison with the 29,431 protein-coding genes estimated for the Phalaenopsis equestris genome (Cai et al. 2015), our gene set for Phalaenopsis 'KHM190' contained 11,722 more members, suggesting a more wider representation of genes in this work. This difference in gene number may be due to different approaches between Phalaenopsis 'KHM190' and Phalaenopsis equestris. Besides, Phalaenopsis 'KHM190' is a hybrid while P. equestris species, which may show gene number difference due to different genetic background. To better annotate the Phalaenopsis genome for protein-coding genes, we generated RNA-seq reads obtained from four tissues as well as publically available expressed sequence tags for cross reference. We defined the function of members of these families using Gene ontology (2008), the Kyoto Encyclopedia of Genes and Genomes (Kanehisa et al. 2012) and Pfam protein motifs (Finn et al. 2014) (Figure. 2 and Dataset S3-S5 and Dataset S19).

The genes in the HC (High confidence) and MC (Medium Confidence) gene sets were functionally annotated based on homology to annotated genes from the NCBI non-redundant database (Dataset S3). The functional domains of Phalaenopsis genes were identified by comparing their sequences against protein databases, including the Gene Ontology (GO) (2008), Kyoto Encyclopedia of Genes and Genomes (KEGG) (Kanehisa et al. 2012) and Pfam (Finn et al. 2014; Finn et al. 2011) databases. GO terms were obtained using the Blast2GO program (Conesa \& Gotz 2008). In the GO annotations, 16,034, 27,294, and 16,360 genes were assigned to the biological process, cellular compound, and molecular function categories, respectively (Figure. 2A). Based on KEGG pathway mapping, we were able to assign a significant proportion of the Phalaenopsis gene sets to KEGG functional or biological pathway categories (11,452 sequences; 140 KEGG orthologous terms) (Dataset S4). To investigate protein families, we compared the Pfam domains of Phalaenopsis genome. A total of 1,842 Pfam domains were detected among the Phalaenopsis sequences. The most abundant protein domains in Phalaenopsis genome were 
pentatricopeptide repeats (PPRs, pfam01535), followed by the WD40 (pfam00400), EF hand (pfam00036) and ERM (Ezrin/radixin/moesin, pfam00769) domains (Figure. 2B and Dataset S5). Furthermore, conserved domains could be identified in $50.17 \%$ of the predicted protein sequences based on comparison against Pfam databases. In addition, we identified 2,610 transcription factors (6.34\% of the total genes) and transcriptional regulators in 55 gene families (SI Appendix, Fig. S8-S10 and Dataset S11-S12).

3.3 Regulation of Phalaenopsis floral organ development. The relative expression of all Phalaenopsis genes was compared through RNA-Seq analysis of shoot tip tissues from shortened stems, leaf, floral organs and PLB samples, in addition to vegetative tissues, reproductive tissues, and germinating seeds from $P$. aphrodite (Su et al. 2011; Tsai et al. 2013) (SI Appendix, Fig. S12 and Dataset S1). Phalaenopsis orchids exhibit a unique flower morphology involving outer tepals, lateral inner tepals and a particularly conspicuous labellum (lip) (Rudall \& Bateman 2002). However, our understanding of the regulation of the floral organ development of the genus is still in its infancy. To comprehensively characterize the genes involved in the development of Phalaenopsis floral organs, we obtained RNA-Seq data for the sepals, petals and labellum of both the wild-type and peloric mutant of Phalaenopsis 'KHM190' at the 0.2-cm floral bud stage, at which shows early sign of labellum differentiation. This cultivar presented an early peloric fate in its lateral inner tepals. In a peloric flower, the lateral inner tepals are converted into a lip-like morphology at this young bud stage (SI Appendix, Fig. S12a and 11b). We identified 3,743 genes that were differentially expressed in the floral organs of the wild-type and peloric mutant plants. Gene Ontology analysis of the differentially expressed genes in Phalaenopsis floral organs revealed functions related to biological regulation, developmental processes and nucleotide binding, which were significantly altered in both genotypes (Huang et al. 2015). Transcription factors (TFs) seem to play a role in floral organ development. Of the 3,309 putative TF genes identified in the Phalaenopsis genome showed differences in expression between the wild-type and peloric mutant plants (Dataset S11).

MADS-box genes are of ancient origin and are found in plants, yeasts and animals (Trobner et al. 1992). This gene family can be divided into two main lineages, referred to as type I and type II. Type I genes only share sequence similarity with type II genes in the MADS domain (Alvarez-Buylla et al. 2000). Most of the well-studied plant genes are type II genes and contain three domains that are not present in type I genes: an intervening (I) domain, a keratin-like coiled-coil (K) domain, and a C-terminal (C) domain (Munster et al. 1997). These genes are best known for their roles in the specification of floral organ development, the regulation of flowering time and other aspects of reproductive development (Dornelas et al. 2011). In addition, MADSbox genes are also widely expressed in vegetative tissues (Messenguy \& Dubois 2003; Parenicova et al. 2003). The ABCDE model comprises five major classes of homeotic selector 
genes: A, B, C, D and E, most of which are MADS-box genes (Theissen 2001). However, research on the $\mathrm{ABCDE}$ model was mainly focused on herbaceous plants and has not fully explained how diverse angiosperms evolved. The function of many other genes expressed during floral development remains obscure. Phalaenopsis exhibits unique flower morphology involving three types of perianth organs: outer tepals, lateral inner tepals, and a labellum (Rudall \& Bateman 2002). Despite its unique floral morphological features, the molecular mechanism of floral development in Phalaenopsis orchid remains largely unclear, and further research is needed to identify genes involved in floral differentiation. Recently, several remarkable research studies on Phalaenopsis MADS-box genes have revealed important roles of some of these genes in floral development, such as four B-class $D E F$-like MADS-box genes that are differentially expressed between wild-type plants and peloric mutants with lip-like petals (Tsai et al. 2004) and a PI-like gene, PeMADS6, that is ubiquitously expressed in petaloid sepals, petals, columns and ovaries (Tsai et al. 2005).

In the Phalaenopsis genome sequence assembly, a total of 122 genes were predicted to encode MADS-box family proteins (SI Appendix, Fig. S8, Dataset S12). To obtain a more accurate classification, phylogenetic trees were constructed via the neighbour-joining method, with 1000 bootstraps using MEGA5 (Tamura et al. 2011).. The differentially expressed genes (DEGs) among 122 Phalaenopsis MADS-box genes were obtained from our Phalaenopsis RNASeq data (Dataset S11). The expression profile indicated that most MADS-box genes are widely expressed in diverse tissues. These results will be helpful to elucidate the regulatory roles of these genes in Phalaenopsis floral organ development.

Notably, we previously reported one of the MADS-box genes, PhAGL6b, upregulated in the peloric lateral inner tepals (lip-like petals) and lip organs (Huang et al. 2015). To understand the expression mode, we therefore cloned the full-length sequence of PhAGL6b from lip organ cDNA libraries for the wild-type, peloric mutant and big lip mutant. The big lip mutant developed a petaloid labellum instead of the regular lip observed in the wild-type flower (Figure 3B). Interestingly, we identified firstly four alternatively spliced forms of PhAGL6b that were specifically expressed only in the petaloid labellum of the big lip mutant (Figure 3C and 3D and SI Appendix, Fig. S11. To determine whether the alternatively spliced forms of PhAGL $6 b$ affect the conversion of the labellum to a petal-like organ in the big lip mutant, we performed RT-PCR of total RNA extracted from the labellum organs of plants with different big lip mutant phenotypes and wild-type plants (SI Appendix, Table S11, Figure 4A) to amplify the PhAGL6b transcripts. Interestingly, among all of the big lip mutant phenotypes, 500 700 bp bands were detected, corresponding to PhAGL6b alternatively spliced forms, which were not found in any of the other orchid plants (Figure 4A). We further examined the expression of PhAGL6b and its alternatively spliced forms in the labellum organs of Phalaenopsis plants with different big lip phenotypes and wild-type plants via real-time PCR (SI Appendix, Table S11). In the big lip 
257

258

259

260

261

262

263

264

265

mutants, the expression of native PhAGL6b was reduced by $42 \sim 70 \%$, whereas all of the alternatively spliced forms were expressed more strongly compared with the wild-type plants (figure 4B). In summary, the RT-PCR and real-time PCR experiments corroborated the specific expression of the alternatively spliced forms of PhAGL6b in the petal-like lip of big lip mutants. Thus, PhAGL6b might play crucial role in the development of the labellum in Phalaenopsis.

The four isoforms of the encoded PhAGL6b products differ only in the length of their Cterminus region (Figure 3D). C-domain is important for the activation of transcription of target genes (Honma \& Goto 2001) and may affect the nature of the interactions with other MADS-box proteins in multimeric complexes (Geuten et al. 2006; Gramzow \& Theissen 2010). In Oncidium, L (lip) complex (OAP3-2/OAGL6-2/OAGL6-2/OPI) is required for lip formation (Hsu et al. 2015). The Phalaenopsis PhAGL6b is an orthologue of OAGL6-2. In our study, the PhAGL6b and its different spliced forms may each other compete the Phalaenopsis L-like complex to affect labellum development as reported in Oncidium (Hsu et al. 2015). This provides a novel clue further supporting the notion that PhAGL6b may function as a key floral organ regulator in Phalaenopsis orchids, with broad impacts on petal, sepal and labellum development (Figure 3E).

3.4 Control of flowering time in Phalaenopsis. The flowering of Phalaenopsis orchids is a response to cues related to seasonal changes in light (Wang 1995), temperature (Blanchard \& Runkle 2006) and other external influences (Chen et al. 1994). A cool night temperature of 18$20^{\circ} \mathrm{C}$ for approximately 4 weeks will generally induce spiking in most Phalaenopsis hybrids, while high temperature inhibits it. To compare gene expression between a constant hightemperature $\left(30 / 27^{\circ} \mathrm{C}\right.$; day/night) and inducing cool temperature $\left(22 / 18^{\circ} \mathrm{C}\right)$, we collected shoot tip tissues from shortened stems of mature $P$. aphrodite plants after treatment at a constant high temperature $(\mathrm{BH})$ and a cool temperature $(\mathrm{BL})$ (1 to 4 weeks) for RNA-Seq data analysis (SI Appendix, Fig. S12g-i). More than 7,500 Phalaenopsis genes were found to be highly expressed in the floral meristems during the 4 successive cool temperature periods (showing at least a 2-fold difference in the expression level in the $\mathrm{BL}$ condition relative to $\mathrm{BH}$ ) (Dataset $\mathrm{S} 13$ ). The identified flowering-related genes correspond to transcription factors and genes involved in signal transduction, development and metabolism (Figure 3 and Dataset S14). The classification of these genes includes the following categories: photoperiod, gibberellins (GAs), ambient temperature, light-quality pathways, autonomous pathways and floral pathway integrators (Fornara et al. 2010; Mouradov et al. 2002). However, the genes involved in the photoperiod, ambient temperature, light quality and autonomous pathways did not show significant changes in the floral meristems during the cool temperature treatments (SI Appendix, Fig. S13 and Dataset S14). By contrast, the expression patterns of genes involved in pathways that regulate flowering, comprising a total of 22 GA pathway-related genes, were related to biosynthesis, signal transduction and responsiveness. The GA pathway-related genes and the floral pathway integrator 
294

295

296

297

298

299

300

301

302

303

304

305

306

genes have been revealed as representative key players in the link between flowering promotion pathways and the floral transition regulation network in several plant species (Mutasa-Gottgens \& Hedden 2009). In contrast to the expression patterns observed in BL and $\mathrm{BH}$, the GA biosynthetic pathway and positively acting regulator genes showed high expression levels in BL. Furthermore, the expression level of negatively acting regulators, like DELLA genes identified, was suppressed by the cool temperature which allowing the activation of flowering related genes - The genes included in the flowering promotion pathways and floral pathway integrators were generally upregulated in BL (Figure 5 and Figure 6 and Dataset S11). These findings suggest that the GA pathway may play a crucial role in the regulation of flowering time in Phalaenopsis orchid during cool temperature.

3.5 Genetic polymorphisms for Phalaenopsis orchids. The Phalaenopsis genome assembly also provides the basis for the development of molecular marker-assisted breeding. Analysis of the Phalaenopsis genome revealed a total of 532,285 simple sequence repeats (SSRs) (SI Appendix, Fig. S14 and Table S9 and Dataset S15). To enable the identification of single nucleotide polymorphisms (SNPs), we re-sequenced the genome of a summer flowering species, P. pulcherrima 'B8802', with about tenfolds coverage. Comparison of the genome data from the two Phalaenopsis accessions (KHM190 and B8802) allowed the discovery of 691,532 SNPs, which should be valuable for future development of SNP markers for Phalaenopsis markerassisted selection. (SI Appendix, Fig. S15 and Table S10 and Dataset S16). P. pulcherrima is an important parent for small flower and summer-flowering cultivars in breeding program. These SNP markers may contribute valuable tools for varietal identification, genetic linkage map development, genetic diversity analysis, and marker-assisted selection breeding in Phalaenopsis orchid.

\section{Conclusion}

In this study, we sequenced, de novo assembled, and extensively annotated the genome of one of the most important Phalaenopsis hybrid. We also annotated the genome with a wealth of RNAseq and sRNA-seq from different tissues, and many genes and miRNAs related to floral organ development, flowering time and protocorm (embryo) development were identified. Importantly, this RNA-Seq and sRNA-seq data allowed us to further improve the genome annotation quality. In addition, mining of SSR and SNP molecular markers from the genome and transcriptomes is currently being adopted in advanced breeding programs and comparative genetic studies, which should contribute to efficient Phalaenopsis cultivar development. Despite the P. equestris genome has been reported recently (Cai et al. 2015), focus on floral organ development and flowering time regulation has not been dealt with. In our study, we obtained transcriptomes from shortened stems, which initiate spikes in response to low ambient temperature, and floral organs 
331 and generated valuable data of potentially regulate flowering time key genes and floral organ 332 development. The genome and transcriptome information of our work should provide a 333 constructive reference resource to upgrade the efficiency of cultivation and genetic improvement 334 of Phalaenopsis orchids.

335

336

337

338

339

340

341

342

343

344

345

346

347

348

349

350

351

352

353

354

355

356

\section{Data deposition:}

The Phalaenopsis genome assembly, transcriptomic and sRNA-seq data were deposited in Genbank with BioProject ID PRJNA271641. The version described in this paper is the first version, JXCR00000000. All short-read data are available via Sequence Read Archive: SRR1747138， SRR1753943， SRR1753944， SRR1753945， SRR1753946， SRR1753947, SRR1753948， SRR1753949， SRR1753950， SRR1752971， SRR1753106， SRR1753165, SRR1753166 (Phalaenopsis 'KHM190' genomic DNA); SRR1762751, SRR1762752, SRR1762753 (Phalaenopsis 'B8802' genomic DNA); SRR1760428, SRR1760429, SRR1760430， SRR1760432， SRR1760433， SRR1760435， SRR1760436， SRR1760438, SRR1760439, SRX396172, SRX396784, SRX396785, SRX396786, SRX396787, SRX396788 (RNA-seq); SRR1760091， SRR1760211， SRR1760212， SRR1760213， SRR1760270， SRR1760271， SRR1760523， SRR1760524， SRR1760525， SRR1760526， SRR1760527, SRR1760528, SRR1760530, SRR1760531, SRR1760532 (small RNA) 
357 Figure Legends

358 Figure 1. Venn diagram showing unique and shared gene families between and among

359 Phalaenopsis, Oryza, Arabidopsis and Vitis.

Figure 2. GO (A) and Pfam (B) annotation of Phalaenopsis protein-coding genes.

Figure 3. Possible evolutionary relationship of $P h A G L 6 b$ in the regulation of lip formation and floral symmetry in Phalaenopsis orchid. Representative RT-PCR result showing the mRNA splicing pattern of PhAGL6b in wild-type (W) and big lip mutant (M). (D) Alignment of the amino acid sequences of alternatively spliced forms of PhAGL6b. (E) Model of PhAGL6b spatial expression for controlling Phalaenopsis floral symmetry. Ectopic expression of PhAGL6b in the distal domain (petal; pink), petal converts into a lip-like structure that leads to radial symmetry. Ectopic expression in proximal domain, (sepal; blue) sepal converts into a lip-like structure that leads to bilateral symmetry ${ }^{15}$. The alternative processing of PhAGL6b transcripts produced in proximal domain (labellum; pink), labellum converts into a petal-like structure that leads to radial symmetry. PhAGL6b expression patterns in Phalaenopsis floral organs are either an expansion or a reduction across labellum. This implies that PhAGL6b may be a key regulator to the bilateral or radially symmetrical evolvements. Pink color: 2nd whorl of the flower; blue color: 1st whorl of the flower; fan-shaped symbol: petal or petal-like structure; triangle symbol: labellum or lip-like structure; Curved symbol: sepal.

Figure 4. Different labellum types of wild-type and big lip mutant Phalaenopsis flowers. RTPCR analysis of the mRNA splicing pattern of PhAGL6b in wild-type plants (98201-WT1 and 98201-WT2) and different big lip mutant types (A). Splicing variants of PhAGL6b, as detected via qRT-PCR in the labellum organ of different big lip mutant types (B).

Figure 5. Expression profiles of genes of flowering time regulation pathway with high temperature and cool temperature treatment. Only the genes with twofold change in expression during cool temperature treatments are revealed

Figure 6. Predicted pathway in the regulation of spike induction in Phalaenopsis. 
394 Red color indicates that the involved genes are more highly expressed in the GA biosynthesis 395 pathway; whereas pink color of gene names indicates their differential expression in the GA 396 response pathway. Blue colors of gene names represent the activation of flower 397 architecture genes. Red arrows show the steps of the GA signaling stage; Pink arrows direct the 398 steps of inflorescence evocation stage; Blue arrows reveal the steps of flower stalk initiation 399 stage. Black arrows indicate the genes downregulated 2X over. GA20ox, GA3ox, GAMYB, FT, SOC1, LFY and $A P 1$ are upregulated 2X over.

401

402

403

404

405

406

407

408

409

410

411

412

413

414

415

416

417

418

419

420

421

422

423

424

425

426

427

428

429

Supplementary files

430 


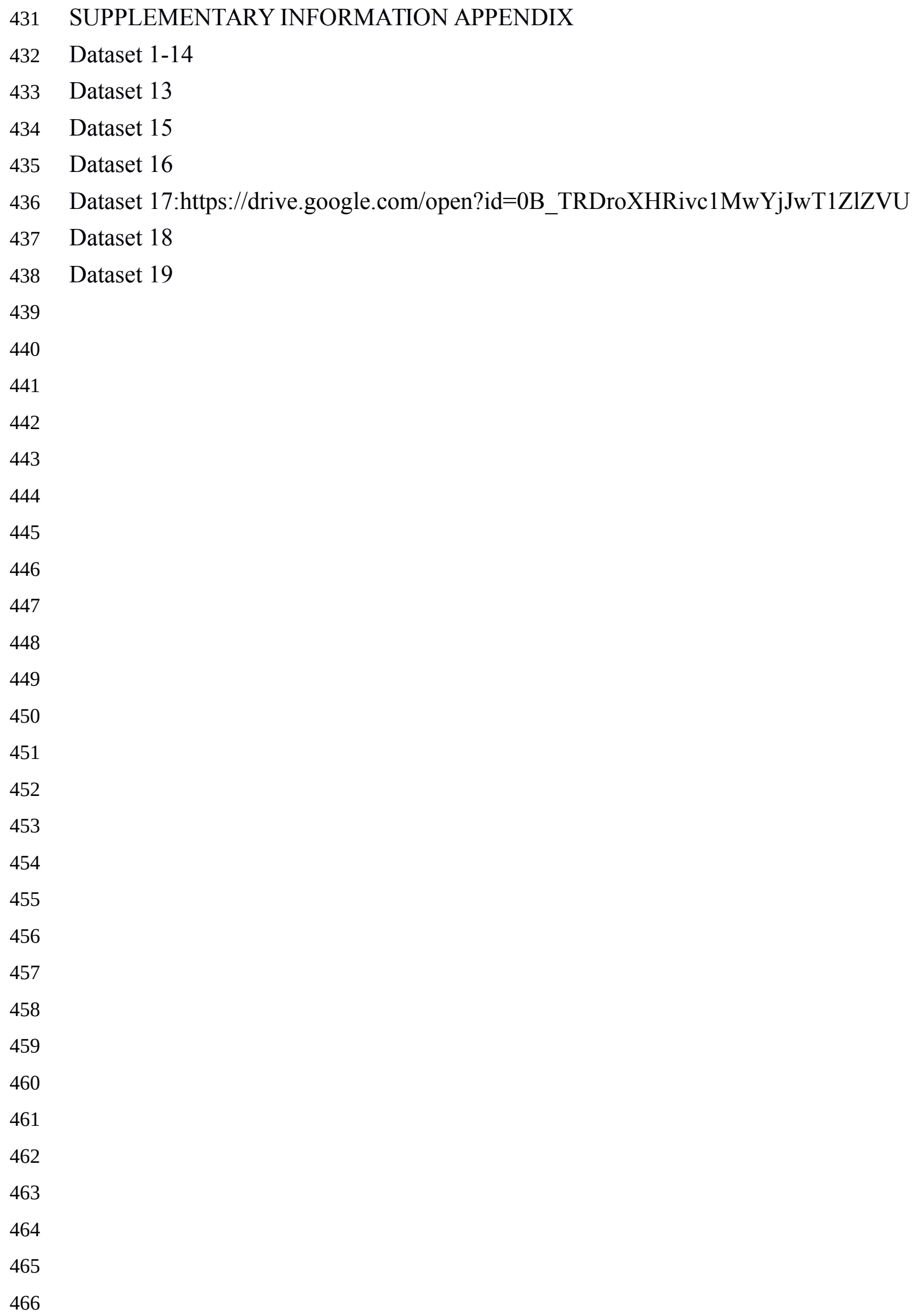




\section{REFERENCES}

2000. Analysis of the genome sequence of the flowering plant Arabidopsis thaliana. Nature 408:796-815. 10.1038/35048692

2005. The map-based sequence of the rice genome. Nature 436:793-800. 10.1038/nature03895

2008. The Gene Ontology project in 2008. Nucleic Acids Res 36:D440-444. 10.1093/nar/gkm883

Alvarez-Buylla ER, Pelaz S, Liljegren SJ, Gold SE, Burgeff C, Ditta GS, Ribas de Pouplana L, Martinez-Castilla L, and Yanofsky MF. 2000. An ancestral MADS-box gene duplication occurred before the divergence of plants and animals. Proc Natl Acad Sci U S A 97:53285333.

An FM, and Chan MT. 2012. Transcriptome-wide characterization of miRNA-directed and nonmiRNA-directed endonucleolytic cleavage using Degradome analysis under low ambient temperature in Phalaenopsis aphrodite subsp. formosana. Plant Cell Physiol 53:17371750. $10.1093 / \mathrm{pcp} / \mathrm{pcs} 118$

An FM, Hsiao SR, and Chan MT. 2011. Sequencing-based approaches reveal low ambient temperature-responsive and tissue-specific microRNAs in phalaenopsis orchid. PLoS One 6:e18937. 10.1371/journal.pone.0018937

Blanchard MG, and Runkle ES. 2006. Temperature during the day, but not during the night, controls flowering of Phalaenopsis orchids. J Exp Bot 57:4043-4049. 10.1093/jxb/erl176

Cai J, Liu X, Vanneste K, Proost S, Tsai WC, Liu KW, Chen LJ, He Y, Xu Q, Bian C, Zheng Z, Sun F, Liu W, Hsiao YY, Pan ZJ, Hsu CC, Yang YP, Hsu YC, Chuang YC, Dievart A, Dufayard JF, Xu X, Wang JY, Wang J, Xiao XJ, Zhao XM, Du R, Zhang GQ, Wang M, Su YY, Xie GC, Liu GH, Li LQ, Huang LQ, Luo YB, Chen HH, Van de Peer Y, and Liu ZJ. 2015. The genome sequence of the orchid Phalaenopsis equestris. Nat Genet 47:65-72. 10.1038/ng.3149

Chang YY, Kao NH, Li JY, Hsu WH, Liang YL, Wu JW, and Yang CH. 2010. Characterization of the possible roles for $\mathrm{B}$ class MADS box genes in regulation of perianth formation in orchid. Plant Physiol 152:837-853. 10.1104/pp.109.147116

Chen W-S, Liu H-Y, Liu Z-H, Yang L, and Chen W-H. 1994. Geibberllin and temperature influence carbohydrate content and flowering in Phalaenopsis. Physiologia Plantarum 90:391-395. 10.1111/j.1399-3054.1994.tb00404.x

Chen WH, Tseng YC, Liu YC, Chuo CM, Chen PT, Tseng KM, Yeh YC, Ger MJ, and Wang HL. 2008. Cool-night temperature induces spike emergence and affects photosynthetic efficiency and metabolizable carbohydrate and organic acid pools in Phalaenopsis aphrodite. Plant Cell Rep 27:1667-1675. 10.1007/s00299-008-0591-0

Christenson EA. 2001. Phalaenopsis: a monograph. Portland Oregon: Timber Press.

Conesa A, and Gotz S. 2008. Blast2GO: A comprehensive suite for functional analysis in plant genomics. Int J Plant Genomics 2008:619832. 10.1155/2008/619832 
504

505

506

507

508

509

510

511

512

513

514

515

516

517

518

519

520

521

522

523

524

525

526

527

528

529

530

531

532

533

534

535

536

537

538

539

540

Dornelas MC, Patreze CM, Angenent GC, and Immink RG. 2011. MADS: the missing link between identity and growth? Trends Plant Sci 16:89-97. 10.1016/j.tplants.2010.11.003

Finn RD, Bateman A, Clements J, Coggill P, Eberhardt RY, Eddy SR, Heger A, Hetherington K, Holm L, Mistry J, Sonnhammer EL, Tate J, and Punta M. 2014. Pfam: the protein families database. Nucleic Acids Res 42:D222-230. 10.1093/nar/gkt1223

Finn RD, Clements J, and Eddy SR. 2011. HMMER web server: interactive sequence similarity searching. Nucleic Acids Res 39:W29-37. 10.1093/nar/gkr367

Fornara F, de Montaigu A, and Coupland G. 2010. SnapShot: Control of flowering in Arabidopsis. Cell 141:550, 550 e551-552. 10.1016/j.cell.2010.04.024

Geuten K, Becker A, Kaufmann K, Caris P, Janssens S, Viaene T, Theissen G, and Smets E. 2006. Petaloidy and petal identity MADS-box genes in the balsaminoid genera Impatiens and Marcgravia. Plant J 47:501-518. 10.1111/j.1365-313X.2006.02800.x

Gramzow L, and Theissen G. 2010. A hitchhiker's guide to the MADS world of plants. Genome Biol 11:214. 10.1186/gb-2010-11-6-214

Honma T, and Goto K. 2001. Complexes of MADS-box proteins are sufficient to convert leaves into floral organs. Nature 409:525-529. 10.1038/35054083

Hsiao YY, Chen YW, Huang SC, Pan ZJ, Fu CH, Chen WH, Tsai WC, and Chen HH. 2011. Gene discovery using next-generation pyrosequencing to develop ESTs for Phalaenopsis orchids. BMC Genomics 12:360. 10.1186/1471-2164-12-360

Hsu H-F, Hsu W-H, Lee Y-I, Mao W-T, Yang J-Y, Li J-Y, and Yang C-H. 2015. Model for perianth formation in orchids. Nature Plants 1. 10.1038/nplants.2015.46

http://www.nature.com/articles/nplants201546\#supplementary-information

Huang JZ, Lin CP, Cheng TC, Chang BC, Cheng SY, Chen YW, Lee CY, Chin SW, and Chen FC. 2015. A de novo floral transcriptome reveals clues into Phalaenopsis orchid flower development. PLoS One 10:e0123474. 10.1371/journal.pone.0123474

Jaillon O, Aury JM, Noel B, Policriti A, Clepet C, Casagrande A, Choisne N, Aubourg S, Vitulo N, Jubin C, Vezzi A, Legeai F, Hugueney P, Dasilva C, Horner D, Mica E, Jublot D, Poulain J, Bruyere C, Billault A, Segurens B, Gouyvenoux M, Ugarte E, Cattonaro F, Anthouard V, Vico V, Del Fabbro C, Alaux M, Di Gaspero G, Dumas V, Felice N, Paillard S, Juman I, Moroldo M, Scalabrin S, Canaguier A, Le Clainche I, Malacrida G, Durand E, Pesole G, Laucou V, Chatelet P, Merdinoglu D, Delledonne M, Pezzotti M, Lecharny A, Scarpelli C, Artiguenave F, Pe ME, Valle G, Morgante M, Caboche M, Adam-Blondon AF, Weissenbach J, Quetier F, and Wincker P. 2007. The grapevine genome sequence suggests ancestral hexaploidization in major angiosperm phyla. Nature 449:463-467. 10.1038/nature06148

Kanehisa M, Goto S, Sato Y, Furumichi M, and Tanabe M. 2012. KEGG for integration and interpretation of large-scale molecular data sets. Nucleic Acids Res 40:D109-114. 
541

542

543

544

545

546

547

548

549

550

551

552

553

554

555

556

557

558

559

560

561

562

563

564

565

566

567

568

569

570

571

572

573

574

575

576

577

\subsection{3/nar/gkr988}

Li L, Stoeckert CJ, Jr., and Roos DS. 2003. OrthoMCL: identification of ortholog groups for eukaryotic genomes. Genome Res 13:2178-2189. 10.1101/gr.1224503

Lin S, Lee HC, Chen WH, Chen CC, Kao YY, Fu YM, Chen YH, Lin TY. 2001. Nuclear DNA contents of Phalaenopsis sp. and Doritis pulcherrima. J Amer Soc Hort Sc.i126: 195-199.

Messenguy F, and Dubois E. 2003. Role of MADS box proteins and their cofactors in combinatorial control of gene expression and cell development. Gene 316:1-21.

Mondragon-Palomino M, and Theissen G. 2011. Conserved differential expression of paralogous DEFICIENS- and GLOBOSA-like MADS-box genes in the flowers of Orchidaceae: refining the 'orchid code'. Plant J 66:1008-1019. 10.1111/j.1365-313X.2011.04560.x

Mouradov A, Cremer F, and Coupland G. 2002. Control of flowering time: interacting pathways as a basis for diversity. Plant Cell 14 Suppl:S111-130.

Munster T, Pahnke J, Di Rosa A, Kim JT, Martin W, Saedler H, and Theissen G. 1997. Floral homeotic genes were recruited from homologous MADS-box genes preexisting in the common ancestor of ferns and seed plants. Proc Natl Acad Sci U S A 94:2415-2420.

Mutasa-Gottgens E, and Hedden P. 2009. Gibberellin as a factor in floral regulatory networks. $J$ Exp Bot 60:1979-1989. 10.1093/jxb/erp040

Parenicova L, de Folter S, Kieffer M, Horner DS, Favalli C, Busscher J, Cook HE, Ingram RM, Kater MM, Davies B, Angenent GC, and Colombo L. 2003. Molecular and phylogenetic analyses of the complete MADS-box transcription factor family in Arabidopsis: new openings to the MADS world. Plant Cell 15:1538-1551.

Rudall PJ, and Bateman RM. 2002. Roles of synorganisation, zygomorphy and heterotopy in floral evolution: the gynostemium and labellum of orchids and other lilioid monocots. Biol Rev Camb Philos Soc 77:403-441.

Su CL, Chao YT, Alex Chang YC, Chen WC, Chen CY, Lee AY, Hwa KT, and Shih MC. 2011. De novo assembly of expressed transcripts and global analysis of the Phalaenopsis aphrodite transcriptome. Plant Cell Physiol 52:1501-1514. 10.1093/pcp/pcr097

Su CL, Chen WC, Lee AY, Chen CY, Chang YC, Chao YT, and Shih MC. 2013. A modified ABCDE model of flowering in orchids based on gene expression profiling studies of the moth orchid Phalaenopsis aphrodite. PLoS One 8:e80462. 10.1371/journal.pone.0080462

Tamura K, Peterson D, Peterson N, Stecher G, Nei M, and Kumar S. 2011. MEGA5: molecular evolutionary genetics analysis using maximum likelihood, evolutionary distance, and maximum parsimony methods. Mol Biol Evol 28:2731-2739. 10.1093/molbev/msr121

Theissen G. 2001. Development of floral organ identity: stories from the MADS house. Curr Opin Plant Biol 4:75-85.

Trapnell C, Pachter L, and Salzberg SL. 2009. TopHat: discovering splice junctions with RNASeq. Bioinformatics 25:1105-1111. 10.1093/bioinformatics/btp120 
578 Trapnell C, Roberts A, Goff L, Pertea G, Kim D, Kelley DR, Pimentel H, Salzberg SL, Rinn JL, and Pachter L. 2012. Differential gene and transcript expression analysis of RNA-seq experiments with TopHat and Cufflinks. Nat Protoc 7:562-578. 10.1038/nprot.2012.016

Trobner W, Ramirez L, Motte P, Hue I, Huijser P, Lonnig WE, Saedler H, Sommer H, and Schwarz-Sommer Z. 1992. GLOBOSA: a homeotic gene which interacts with DEFICIENS in the control of Antirrhinum floral organogenesis. EMBO J 11:4693-4704.

Tsai WC, Fu CH, Hsiao YY, Huang YM, Chen LJ, Wang M, Liu ZJ, and Chen HH. 2013. OrchidBase 2.0: comprehensive collection of Orchidaceae floral transcriptomes. Plant Cell Physiol 54:e7. 10.1093/pcp/pcs187

Tsai WC, Kuoh CS, Chuang MH, Chen WH, and Chen HH. 2004. Four DEF-like MADS box genes displayed distinct floral morphogenetic roles in Phalaenopsis orchid. Plant Cell Physiol 45:831-844. 10.1093/pcp/pch095

Tsai WC, Lee PF, Chen HI, Hsiao YY, Wei WJ, Pan ZJ, Chuang MH, Kuoh CS, Chen WH, and Chen HH. 2005. PeMADS6, a GLOBOSA/PISTILLATA-like gene in Phalaenopsis equestris involved in petaloid formation, and correlated with flower longevity and ovary development. Plant Cell Physiol 46:1125-1139. 10.1093/pcp/pci125

Wang Y-T. 1995. Phalaenopsis Orchid Light Requirement during the Induction of Spiking. HortScience 30:59-61.

Zerbino DR, and Birney E. 2008. Velvet: algorithms for de novo short read assembly using de Bruijn graphs. Genome Res 18:821-829. 10.1101/gr.074492.107 


\section{Figure 1 (on next page)}

Figure 1

Figure 1. Venn diagram showing unique and shared gene families between and among Phalaenopsis, Oryza, Arabidopsis and Vitis. 


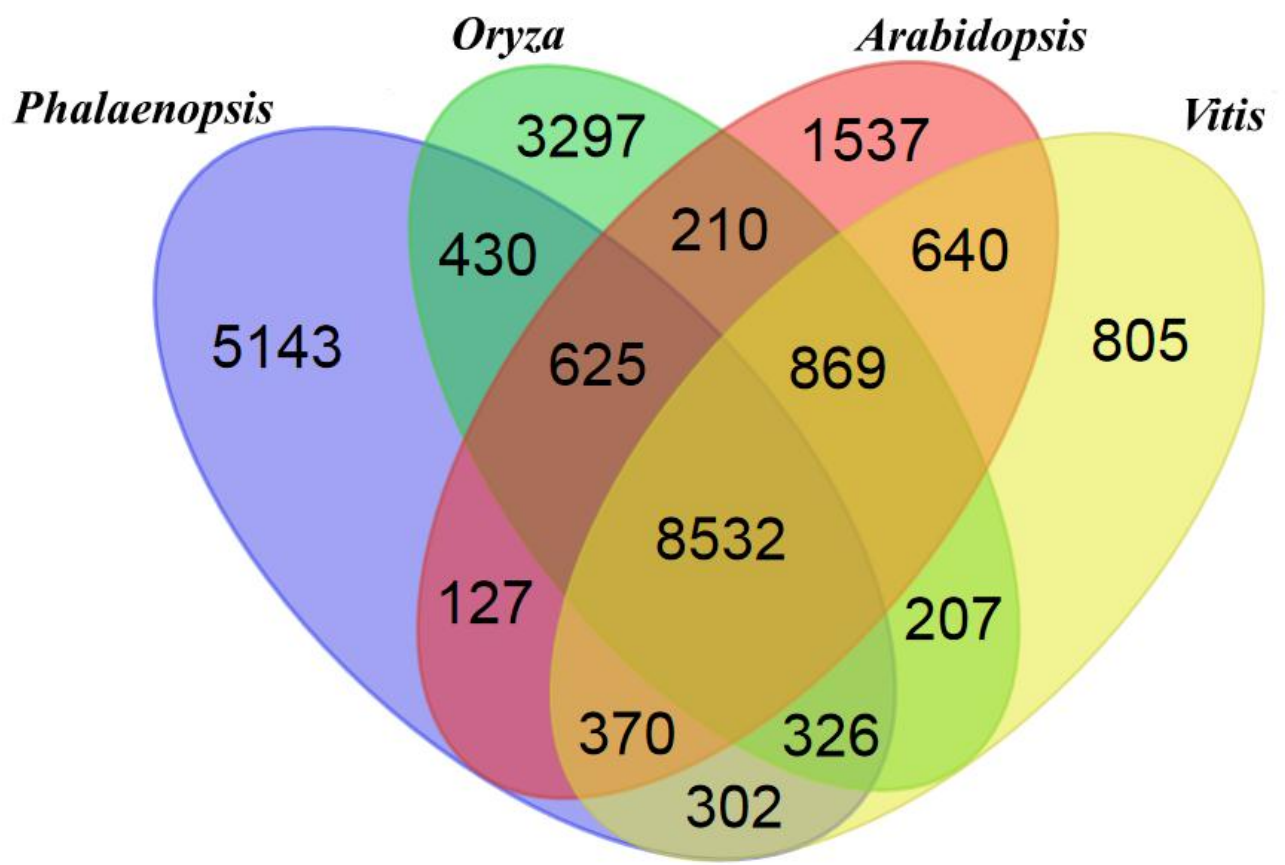




\section{Figure 2 (on next page)}

Figure 2

Figure 2. GO (A) and Pfam (B) annotation of Phalaenopsis protein-coding genes 
A

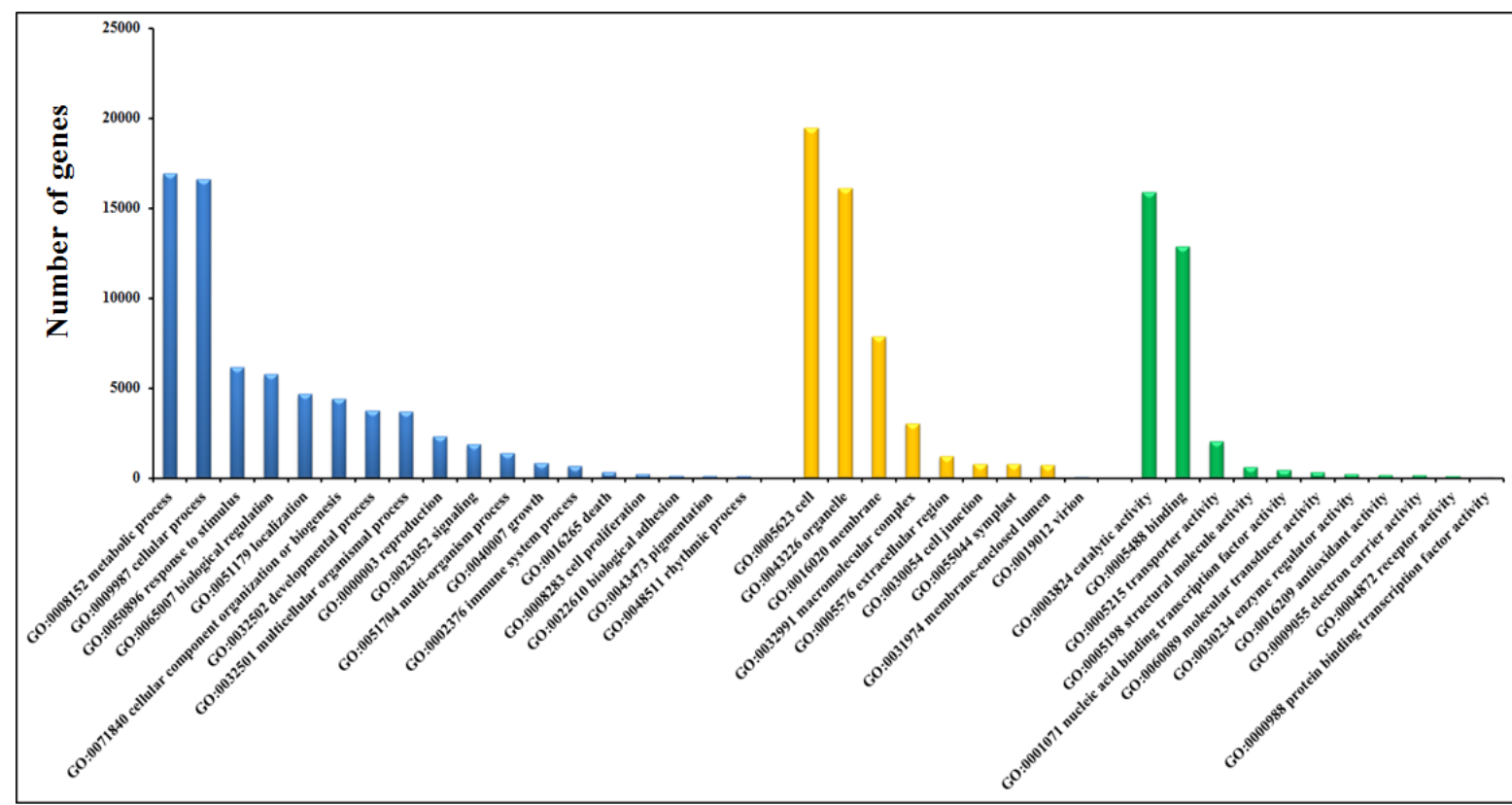

Biological process

Cellular component

Molecular function

B

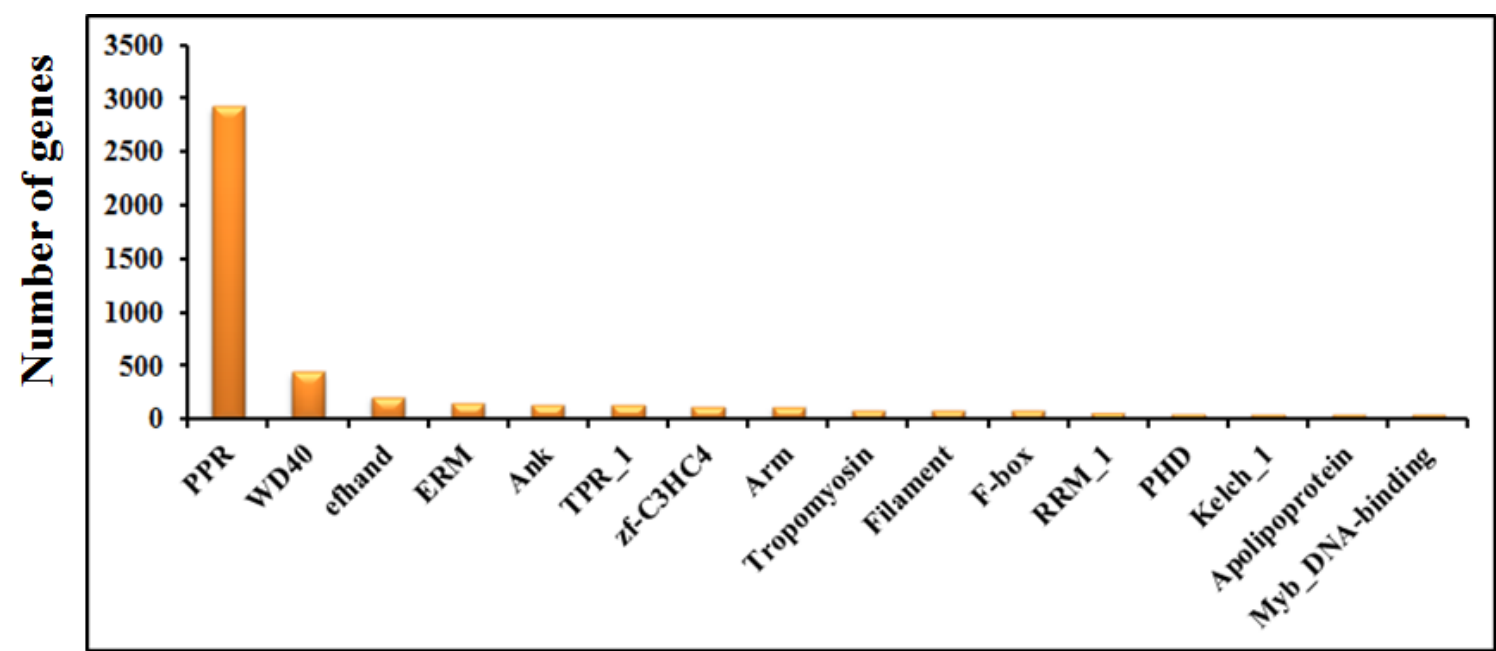

Domain 


\section{Figure 3 (on next page)}

Figure 3 

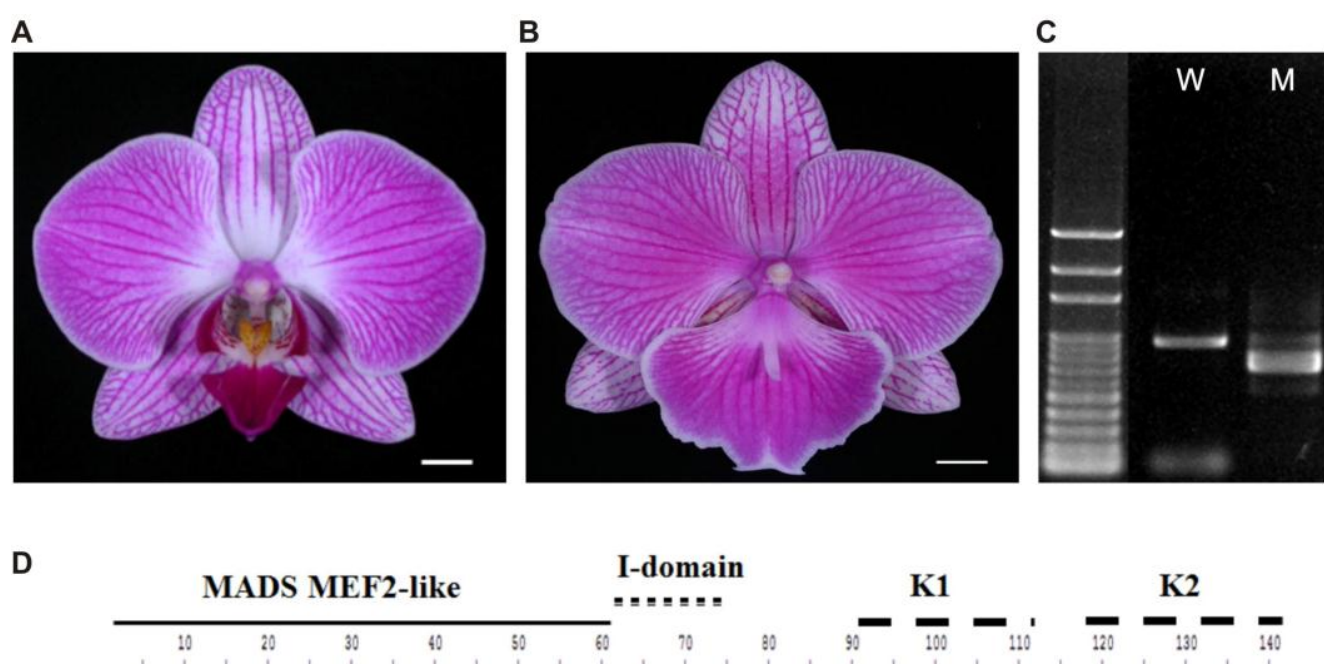

PhAGL6:

PhAGLbb-1
PhaGLGb-2

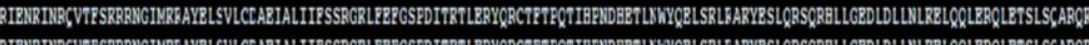

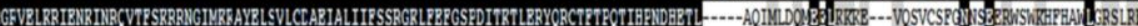

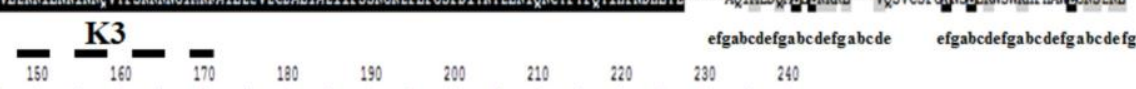

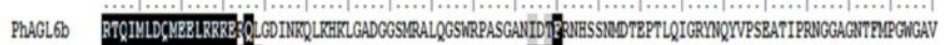

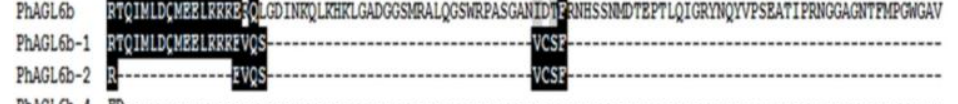

PhAGL6b-2 $\mathrm{B}-\mathrm{O}$

fgabcdefgabcedefgabcetefga --------
E
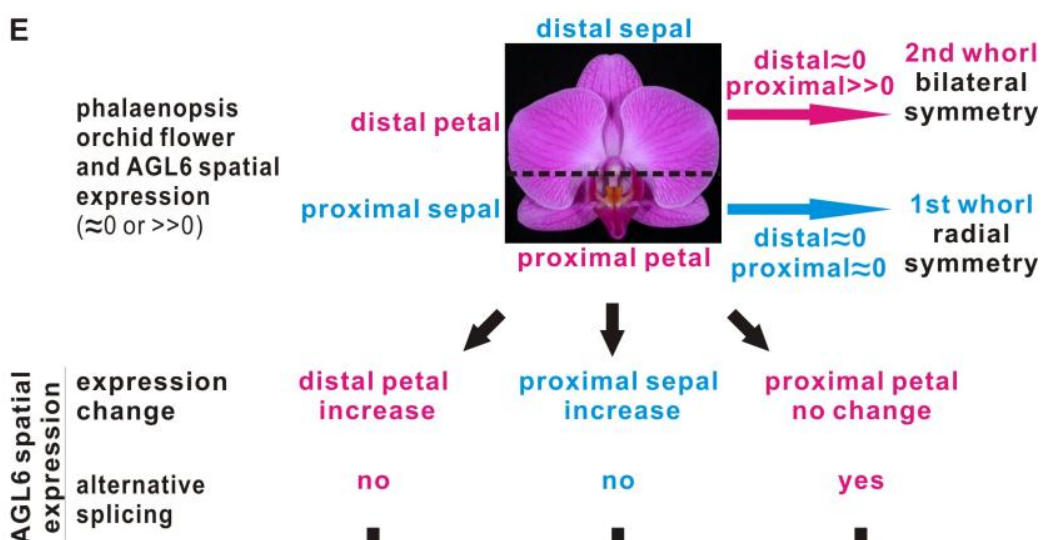
increase

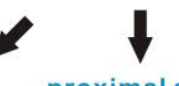

proximal sep

proximal petal
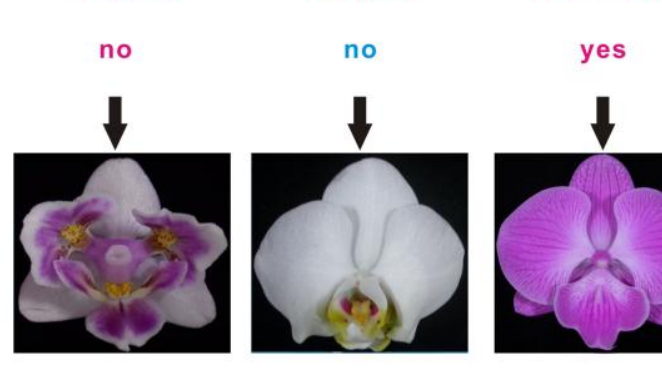

radial

¿ symmetry

AGL6 spatial

के expression

distal $\approx 0$

symmetry

radial

AGL6 spatial

distal $>>0$

proximal $>>0$

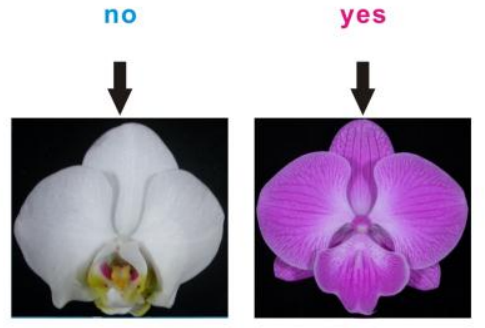

bilateral

distal $\approx 0$

radial

distal $\approx 0$

bilateral

distal $\approx 0$

proximal $>0$ radial

distal $\approx 0$ proximal $>>0$ but AGL6 


\section{Figure 4 (on next page)}

Figure 4

Figure 4. Different labellum types of wild-type and big lip mutant Phalaenopsis flowers. RT-PCR analysis of the mRNA splicing pattern of PhAGL6b in wild-type plants (98201-WT1 and 98201-WT2) and different big lip mutant types (A). Splicing variants of

PhAGL6b, as detected via qRT-PCR in the labellum organ of different big lip mutant types (B). 
A
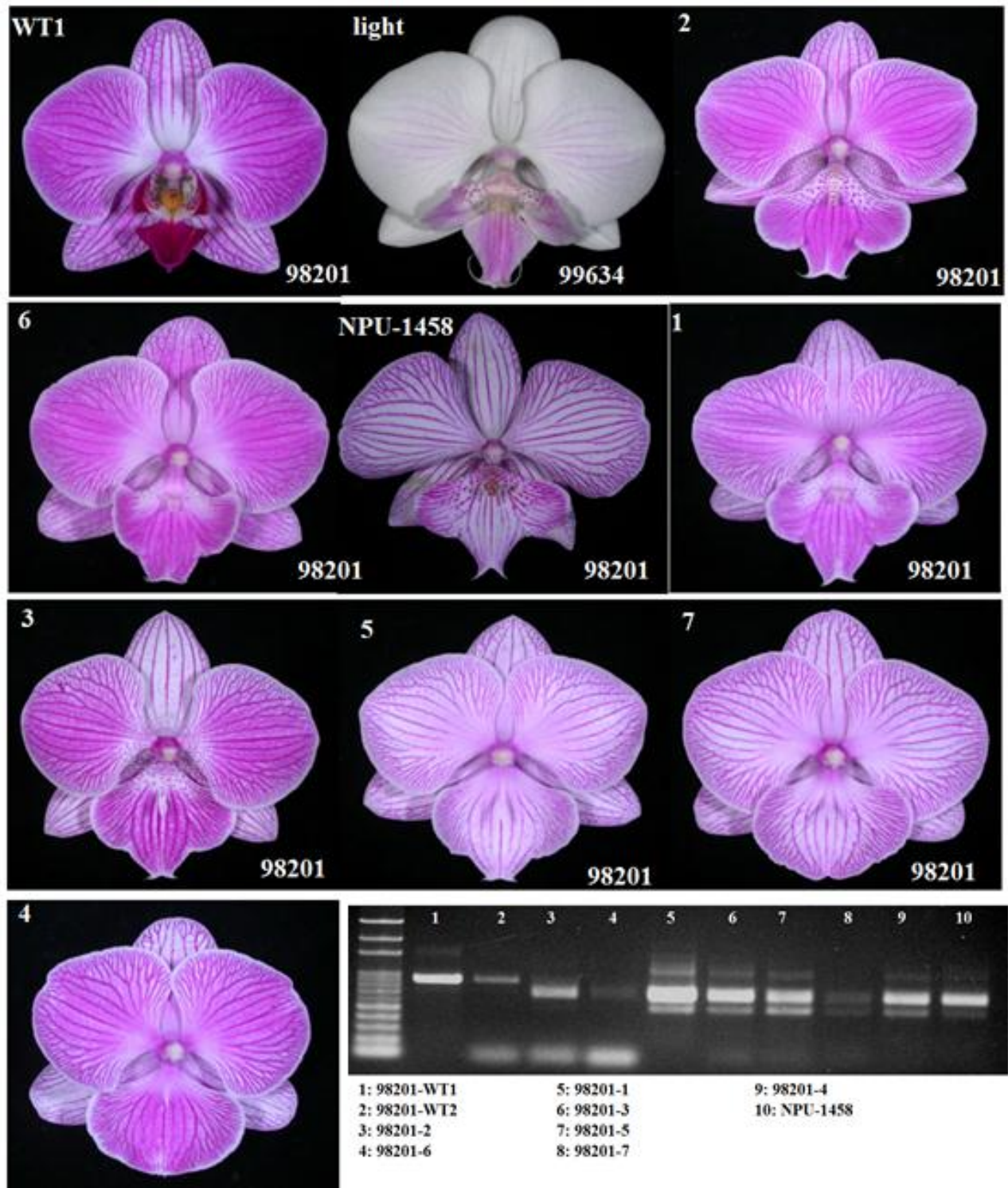

B
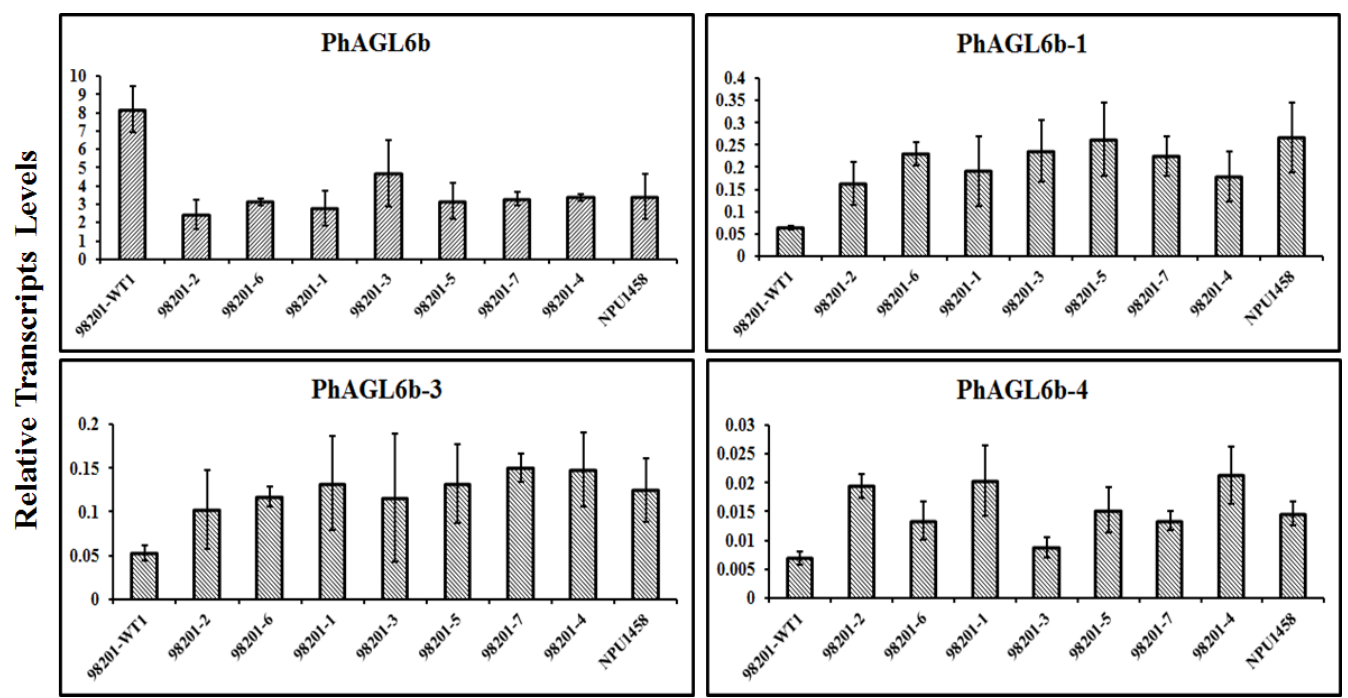


\section{Figure $\mathbf{5}$ (on next page)}

Figure 5 

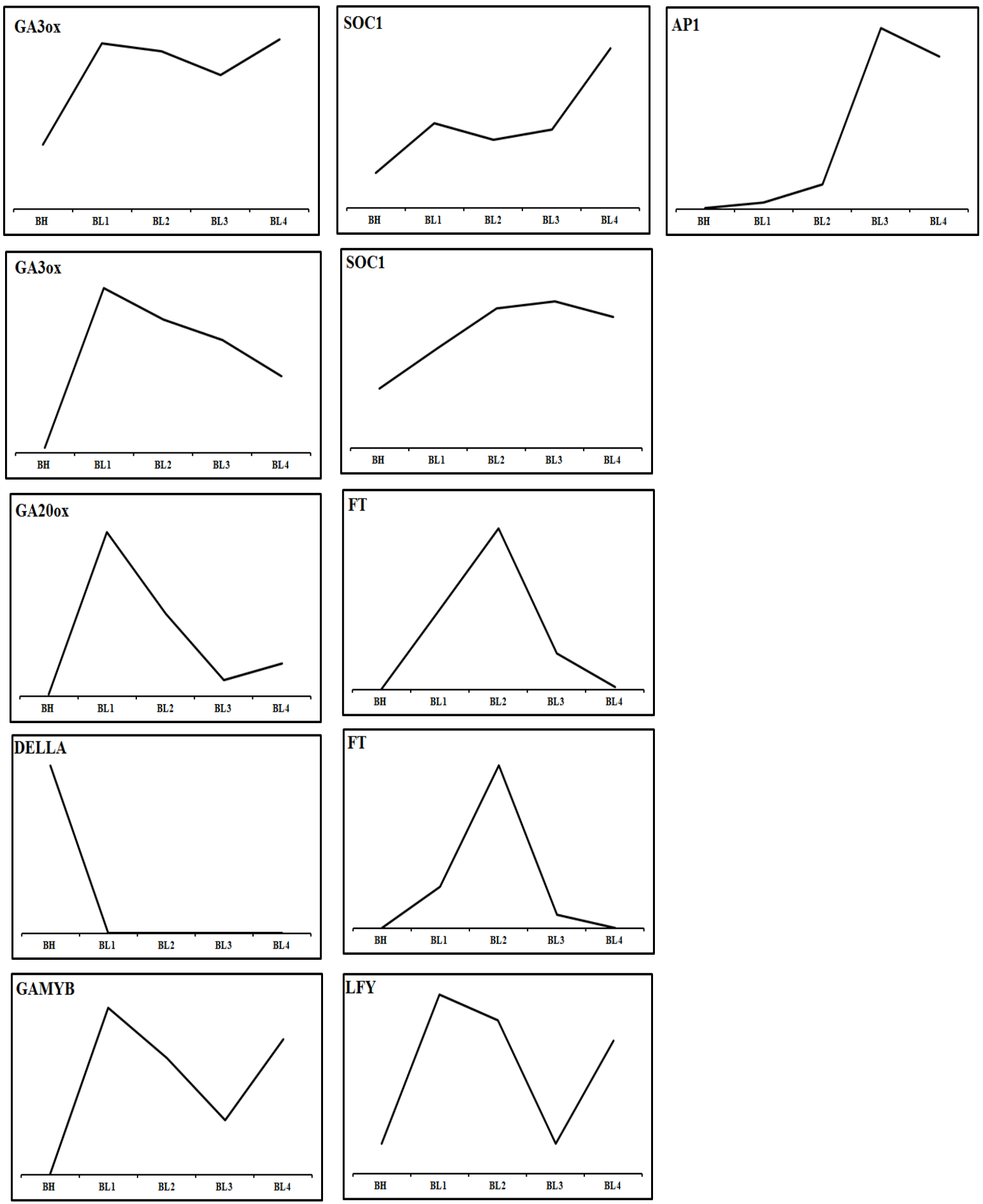
Figure $\mathbf{6}$ (on next page)

Figure 6

Figure 6. Predicted pathway in the regulation of spikeinduction in Phalaenopsis.

Red color indicates that the involved genes are more highly expressed in the GA biosynthesis pathway; whereas pink color of gene names indicates their differential expression in the GA response pathway. Blue colors of gene names represent the activation of flower architecturegenes. Red arrows show the steps ofthe GA signaling stage; Pink arrows direct the steps of inflorescence evocation stage; Blue arrowsrevealthe steps offlower stalk initiation stage. Black arrows indicate the genes downregulated $2 X$ over.GA200X,GA3OX,GAMYB,FT,SOC1, LFYandAP1are upregulated 2X over. 


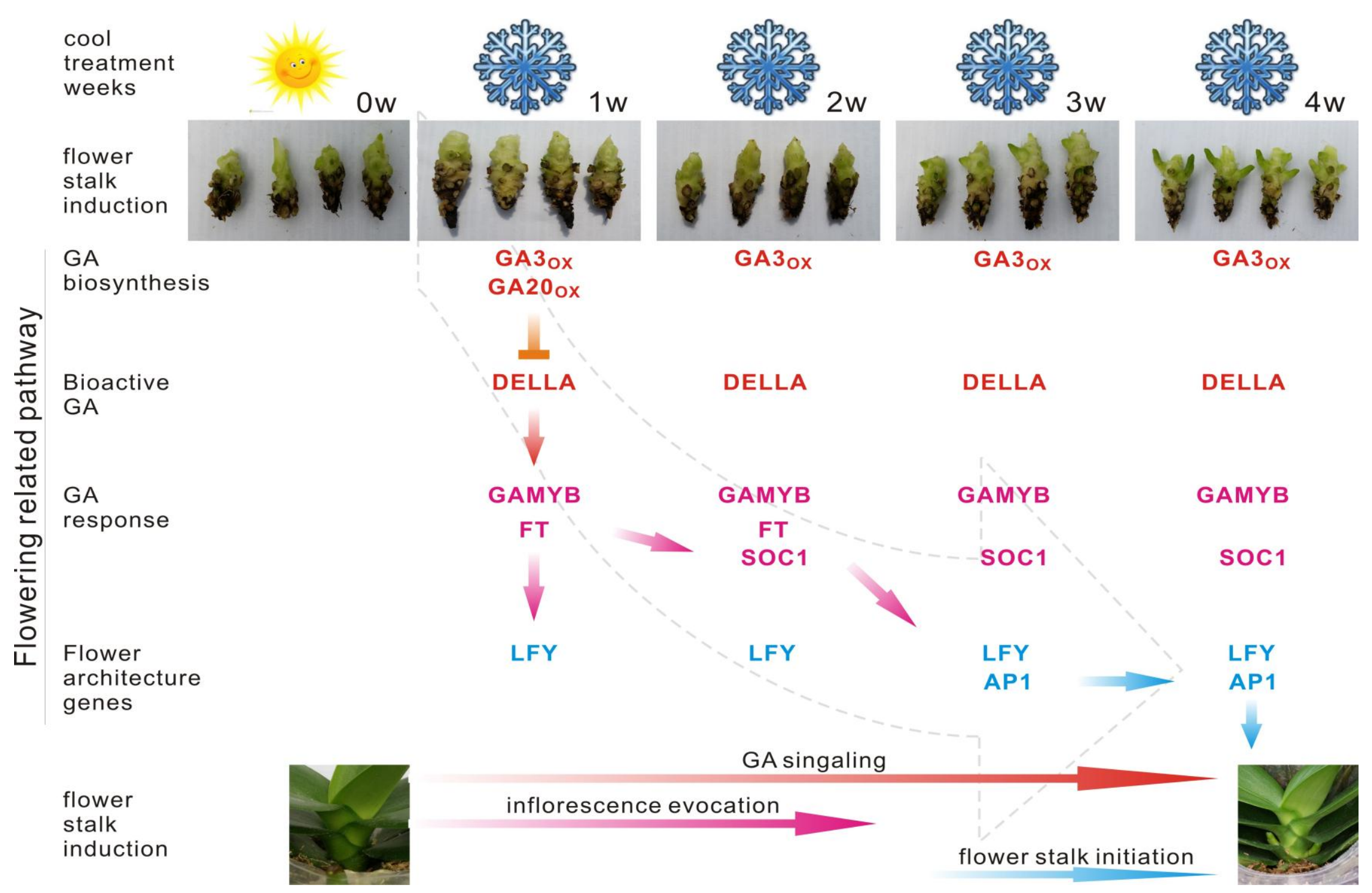




\section{Table $\mathbf{1}$ (on next page)}

Table 1 
1 Table 1 Statistics of the Phalaenopsis draft genome

2 Estimate of genome size

3 Chromosome number (2n)

4 Total size of assembled contigs

5 Number of contigs ( $\geq 1 \mathrm{kp})$

6 Largest contig

$7 \quad$ N50 length (contig)

8 Number of scaffolds ( $\geq 1 \mathrm{kp})$

9 Total size of assembled scaffolds

$10 \quad$ N50 length (scaffolds)

11 Longest scaffold

12 GC content

13 Number of gene models

14 Mean coding sequence length

15 Mean exon length/ number

16 Mean intron length/number

17 Exon GC (\%)

18 Intron GC (\%)

19 Number of predicted miRNA genes

20 Total size of transposable elements
3.45 Gb

38

$3.1 \mathrm{~Gb}$

630,316

50,944

1,489

149,151

3,104,268,398

100,943

$1,402,447$

30.7

41,153

1,014 bp

264 bp / 3.83

3,099 bp / 2.83

41.9

16.1

650

1,598,926,178

21

22

23

24 